\title{
Pénfigo paraneoplásico como presentación atípica de la enfermedad de Castleman
}

\section{Paraneoplastic pemphigus as an atypical presentation of Castleman disease}

\author{
Snayder José Pérez-Goelkel, Caridad Chao-Pereira, Karen Valdés-Álvarez, \\ María Paula Campos-García - La Habana (Cuba).
}

DOI: https://doi.org/10.36104/amc.2021.1964

\section{Resumen}

La enfermedad de Castleman es un trastorno linfoproliferativo no clonal con amplia gama de manifestaciones clínicas. Se presenta el caso de paciente masculino con cuadro clínico consistente en astenia, adinamia, hiporexia, pérdida ponderal, úlceras orales y genitales, prurito ocular e hiperemia conjuntival. El examen físico evidenció hiperemia conjuntival, úlceras orales y genitales. La tomografía axial computarizada contrastada de tórax y abdomen reveló múltiples adenopatías mediastinales, retroperitoneales y masa sólida de 94 x 51 milímetros de localización retroperitoneal. Se realizó biopsia de la masa previamente descrita, que reportó enfermedad de Castleman variante hialino vascular. También se indicó biopsia de lesión escrotal cuyo análisis histopatológico fue compatible con pénfigo, además la fluorescencia inmunológica directa fue positiva en los espacios intercelulares de la epidermis al igual que inmunoprecipitación con anticuerpos anti-desmogleina, anti-desmoplaquina, anti-envoplaquina y antígeno del penfigoide ampollar. Por lo anteriormente descrito se definió la existencia de enfermedad de Castleman multicéntrica asociada a pénfigo paraneoplásico. (Acta Med Colomb 2021; 46. DOI: https://doi.org/10.36104/amc.2021.1964)

Palabras clave: pénfigo, paraneoplásico, Castleman, multicéntrica.

\section{Abstract}

Castleman disease is a non-clonal lymphoproliferative disorder with a broad range of clinical manifestations. We present the case of a male patient with a clinical picture of asthenia, adynamia, hyporexia, weight loss, oral and genital ulcers, and red, itchy eyes. The physical exam showed conjunctival redness and oral and genital ulcers. Computed axial tomography with contrast of the chest and abdomen revealed multiple enlarged mediastinal and retroperitoneal lymph nodes, and a solid 94x51 mm retroperitoneal mass. A biopsy of the mass was taken, which reported the hyaline vascular variant of Castleman disease. A scrotal lesion biopsy was also ordered, with a histopathological analysis compatible with pemphigus. In addition, direct immunofluorescence was positive in the epidermal intercelullar spaces, as well as immunoprecipitation with anti-desmoglein, antidesmoplakin, anti-envoplakin and pemphigoid ampule antigen. Thus, the presence of multicentric Castleman disease associated with paraneoplastic pemphigus was established. (Acta Med Colomb 2021; 46. DOI: https://doi.org/10.36104/amc.2021.1964)

Key words: pemphigus, paraneoplastic, Castleman, multicentric.
Dr. Snayder José Pérez-Goelkel: Especialista en Medicina Interna, Profesor Asistente e Investigador Auxiliar; Dra. Caridad Chao-Pereira: Especialista en Medicina Interna, Doctor en Ciencias Médicas, Profesora Titular e Investigadora Auxiliar; Dra. Karen Valdés-Álvarez: Especialista en Medicina Interna, Master en Infectología, Profesora Asistente e Investigadora Agregada; Dra. María Paula Campos-García: Especialista en Epidemiología, Residente de Medicina Interna. Hospital Hermanos Ameijeiras. La Habana (Cuba).

Correspondencia: Dr. Snayder José Pérez Goelkel. Neiva (Colombia).

E-mail: snayderjose@gmail.com

Recibido 24/VII/2020 Aceptado: 26/VII/ 2021

\section{Introducción}

La enfermedad de Castleman (EC) es un trastorno linfoproliferativo no clonal. Más que una enfermedad constituye un grupo heterogéneo de entidades muy infrecuentes con una amplia gama de manifestaciones clínicas (1). El primer caso se reportó en 1956 dentro de una serie de pacientes con pocos o ningún síntoma, pero con ganglio mediastinal solitario (2). En la EC existen tres variantes mayores reconocidas: hialino vascular (HV), células plasmáticas y virus herpes humano 8 (VHH8) positiva. El informe inicial de Benjamín Castleman corresponde al subtipo HV, donde la arquitectura de los ganglios linfáticos se caracteriza por folículos 
linfoides con centros germinales atróficos o "regresivos" a menudo hialinizados, principalmente constituidos por células dendríticas foliculares residuales y zonas prominentes del manto que contienen pequeños linfocitos. Las células dendríticas foliculares están organizadas en forma concéntrica y proporciona una apariencia de "piel de cebolla". La variante de células plasmáticas es el subtipo más común de la enfermedad multicéntrica (75\%) (3). La coexistencia de células hialovasculares y plasmáticas, son la característica de un subconjunto de EC multicéntrica relacionada con virus herpes humano 8 (4).

En 1990 Anhalt et al. reportaron una entidad clínica llamada "pénfigo paraneoplásico" (PPN), posteriormente Nguyen et al. la describieron como un síndrome autoinmune heterogéneo que afecta a varios órganos internos, ya que su fisiopatología no es limitada a autoanticuerpos dirigidos a moléculas de adhesión. El PPN está estrechamente relacionado con tumores benignos o malignos. Los tumores malignos más frecuentemente reportados son los de tejido linfoide y hematológicos (linfoma de células B, leucemia linfocítica crónica, enfermedad de Castleman, macroglobulinemia de Waldenstrom y timoma) (5). Presentamos el caso de paciente masculino con úlceras orales y genitales en quien se realizó el diagnóstico de PPN como forma atípica de presentación de EC multicéntrica.

\section{Presentación del caso}

Paciente masculino de 52 años de edad quien consultó por cuadro clínico de seis meses de evolución consistente en astenia, adinamia, hiporexia, pérdida ponderal de aproximadamente $18 \mathrm{Kg}$, úlceras dolorosas a nivel bucal y genital, prurito ocular e hiperemia conjuntival, recibió antibioticoterapia empírica con penicilina durante manejo médico en otra institución y posteriormente por sospecha clínica enfermedad de Behçet fue tratado farmacológicamente con prednisona y talidomida, sin lograr remisión de las manifestaciones clínicas. El examen físico evidenció hiperemia conjuntival, úlceras orales sin toma de encías (Figura 1A) y genitales con compromiso de escroto y prepucio (Figura 1B) además varicocele izquierdo no reductible. Los estudios complementarios mostraron: hemograma con trombocitosis en $506 \times 10^{9} / \mathrm{L}$, leucocitos en 6.4 x $10^{9} \mathrm{~g} / \mathrm{L}$ y hemoglobina $150 \mathrm{~g} / \mathrm{L}$. La hemoquímica reportó hiperuricemia $(562 \mu \mathrm{mol} / \mathrm{L})$, eritrosedimentación acelerada ( $80 \mathrm{~mL} / \mathrm{hora})$, proteína $\mathrm{C}$ reactiva elevada en 80 miligramos $(\mathrm{mg}) / \mathrm{L}$, además los resultados de anticuerpos antinucleares, anticuerpos anticitoplasmáticos del neutrófilo, serología para anticuerpos antivirus de herpes humano 8, anticuerpos antivirus de inmunodeficiencia humano tipos 1 y 2 fueron negativos. Recibió valoración por reumatología

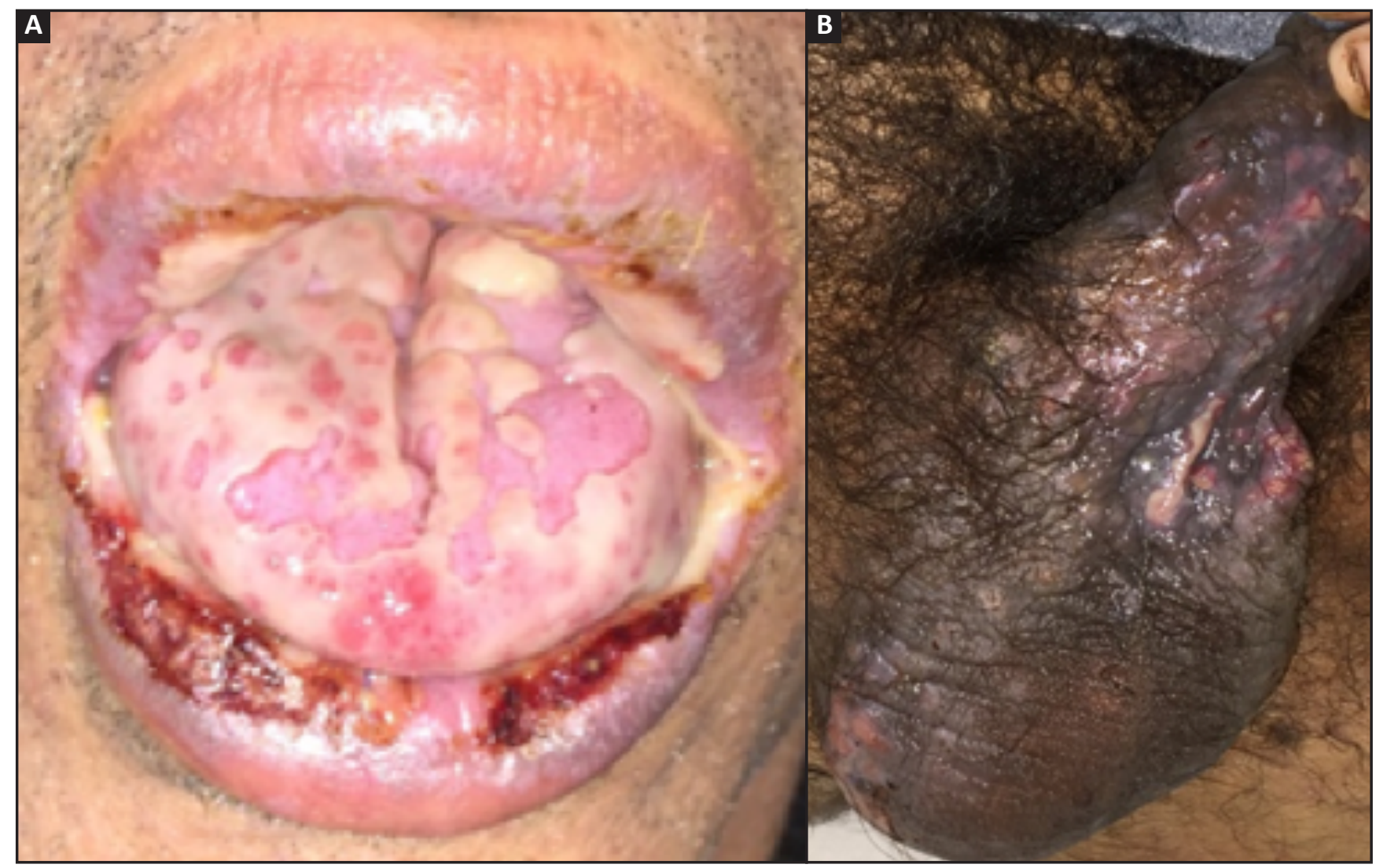

Figura 1. A. Estomatitis severa. Queilitis erosiva con presencia de costras serohemáticas. Lengua con erosiones cubiertas con membranas blanquecinas. B. Lesiones ulceradas cubiertas con membranas blanquecinas a nivel de escroto y prepucio. 
quienes indicaron prueba de patergia reportada negativa y la evaluación oftalmológica no encontró signos uveítis anterior o posterior por lo que se descartó la posibilidad de enfermedad de Behçet. Le fue realizado medulograma y biopsia de médula que no evidenció infiltración tumoral. Estudios imagenológicos: ultrasonografía de abdomen donde fue observada masa de localización retroperitoneal por lo que se procedió a realizar tomografía axial computarizada contrastada de abdomen donde se evidenció masa sólida de 94 x 51 milímetros con densidad heterogénea y múltiples microcalcificaciones, localizada en retroperitoneo, a la derecha de la línea media, que desplazaba ventralmente y comprimía a la vena renal izquierda lo que justificaba el varicocele ipsilateral no reductible encontrado en examen físico, también múltiples adenopatías retroperitoneales vecinas (Figura 2). Se extendió exploración tomográfica hasta el tórax donde encontraron múltiples adenopatías mediastinales posteriores paraesofágicas que se extendían al espacio retrocrural, la mayor de 23 x 34 mm. Se realizó biopsia de masa retroperitoneal guiada por tomografía que reportó enfermedad de Castleman variante HV (Figura 3A) con las siguientes especificidades inmuhistoquímicas: CD3, CD20, KI67, CD21 positivos con BCL6 y CD30 negativos. También se practicó biopsia de lesión cutánea prepucial (Figura 3B) cuyo informe histopatológico describió la pérdida casi total de la epidermis con separación intraepidérmica secundaria a acantólisis, presencia de numerosas células acantolíticas aisladas y agrupadas, de núcleos hipercromáticos y citoplasma homogéneo, queratinocitos necróticos y vacuolización de la basal. Además, se detectó fluorescencia inmunológica en los espacios intercelulares de la epidermis con anticuerpos monoclonales dirigidos contra la inmunoglobulina $\mathrm{G}$ y el factor 3 del complemento. Teniendo en cuenta reporte de histopatología, inmunofluorescencia

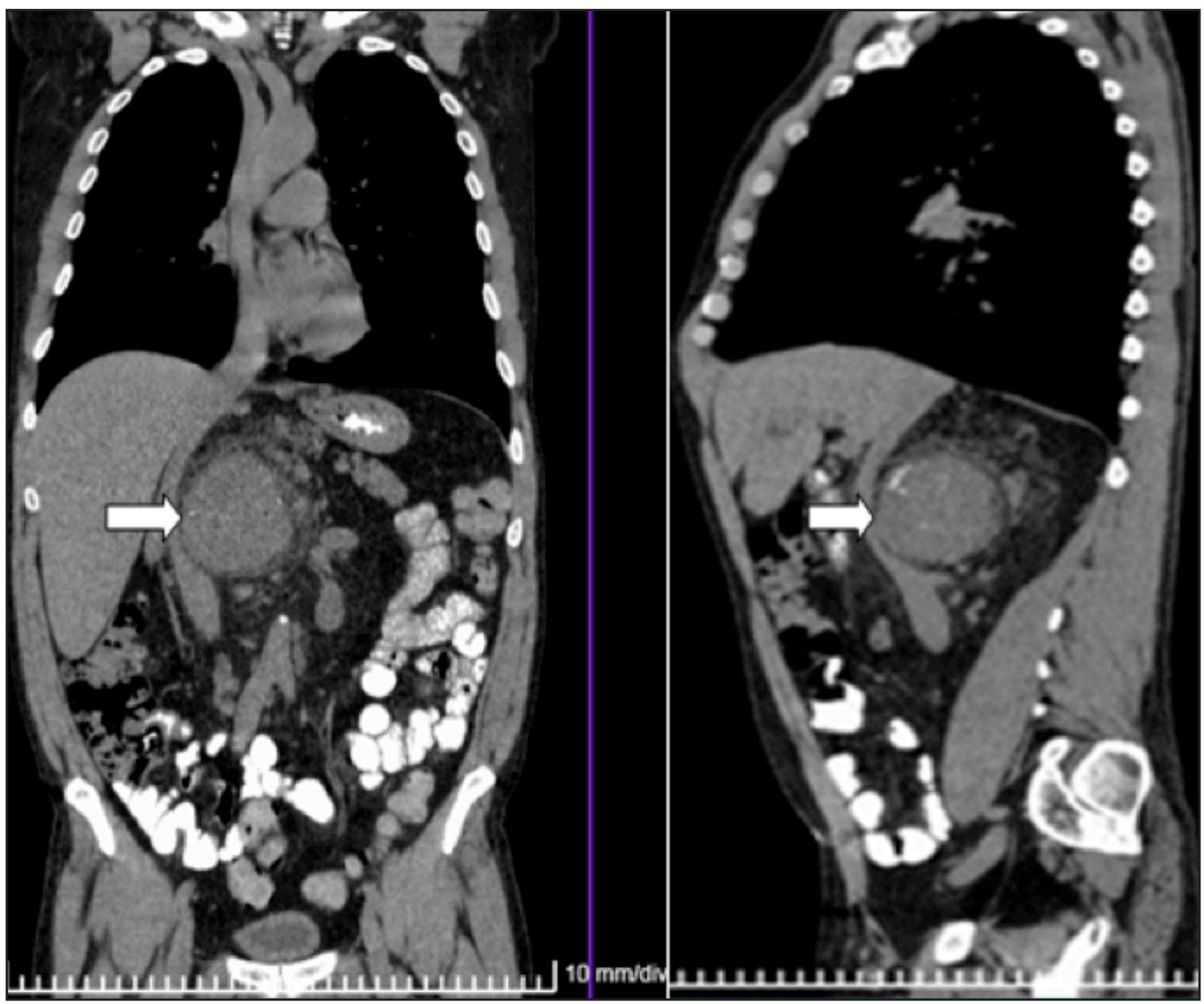

Figura 2. TAC de abdomen. Masa retroperitoneal sólida con densidad heterogénea y múltiples micro calcificaciones a la derecha de la línea media, por enfermedad de Castleman en paciente con pénfigo paraneoplásico. 


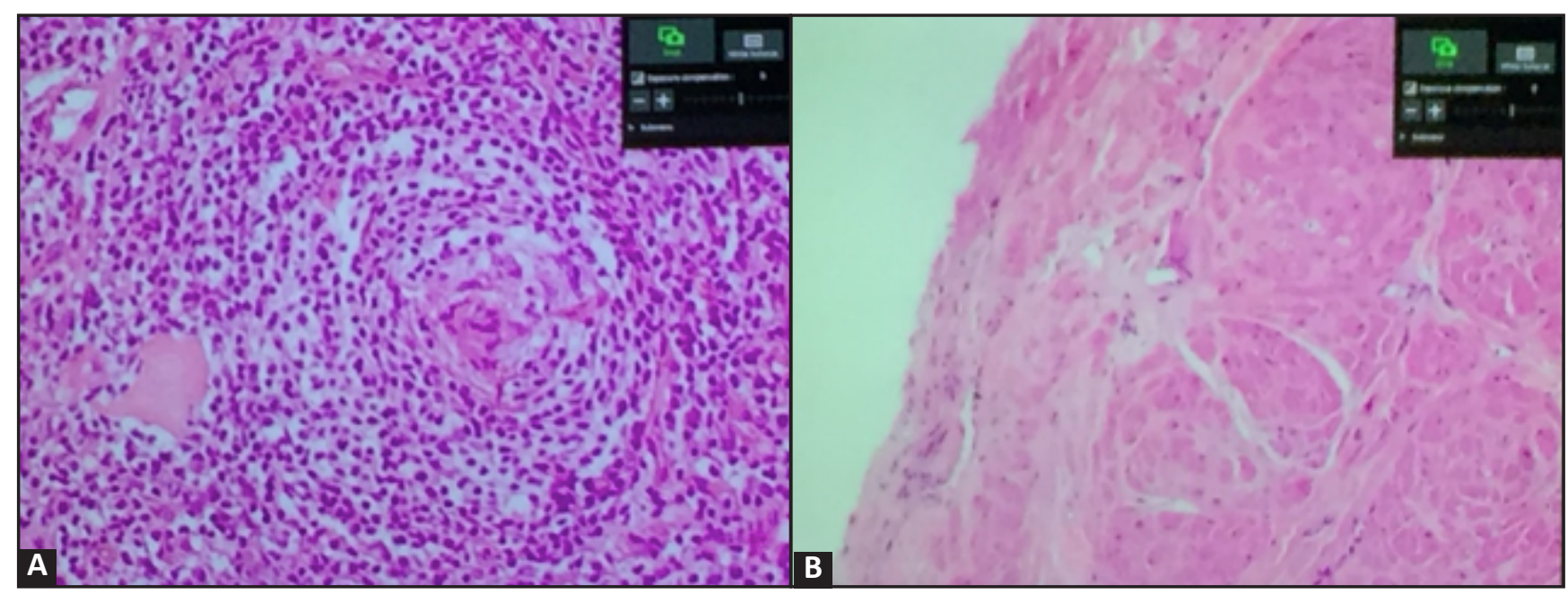

Figura 3. A. Histopatología de masa retroperitoneal que muestra folículos linfoides pequeños con disposición concéntrica de los linfocitos, aspecto de "piel de cebolla" en la hiperplasia angiofolicular compatible con enfermedad de Castleman variante hialino-vascular (Hematoxilina eosina 40x). B. Histopatología de lesión cutánea que muestra pérdida casi total de la epidermis con separación intraepidérmica secundaria a acantólisis y necrosis acompañante compatible con pénfigo (Hematoxilina eosina 40x).

directa positiva y hallazgos macroscópicos en piel descritos previamente, en presencia de EC se sospechó con solidez la existencia de PPN, por lo que se indicaron anticuerpos antidesmogleína 1 y 3 , antidesmoplaquina 1 , antienvoplaquina y antígeno del penfigoide ampollar que resultaron positivos. No fueron realizados los estudios de inmunofluorescencia indirecta en vejiga transicional de rata o epitelio esofágico de mono debido a la falta de disponibilidad, pero basados en los hallazgos clínicos, imagenológicos, inmunológicos y anatomopatológicos previamente descritos se diagnosticó enfermedad de Castleman multicéntrica variante HV asociada a pénfigo paraneoplásico (PPN). Recibió quimioterapia con ciclofosfamida, doxorrubicina, vincristina y prednisona (CHOP) sin obtener remisión parcial o total de las úlceras orales y genitales diagnosticadas como PPN.

\section{Discusión}

La enfermedad de Castleman constituye un raro trastorno linfoproliferativo que fue descrito por primera vez hace más de 60 años como una masa benigna y localizada (6). Desde el punto de vista clínico esta enfermedad ha sido dividida en dos subtipos: EC unicéntrica (ECU) y EC multicéntrica (ECM). Existe un contraste respecto a las manifestaciones clínicas de los subtipos enunciados ya que la ECU habitualmente tiende a ser paucisintomática y su variante histológica más frecuente es la HV que corresponde a 80$90 \%$ de los casos, mientras que la ECM incluye un tipo de células plasmáticas y un tipo mixto, que a menudo causan manifestaciones sistémicas, como fiebre, perdida ponderal, hipoproteinemia, linfadenopatía sistémica, deterioro de la función renal e incluso síndrome POEMS (polirradiculoneuropatía, organomegalia, endocrinopatía, trastorno de proliferación clonal de células plasmáticas y cambios cutáneos) (6-7). Cabe señalar que, la variante histológica HV tiene presentación clínica unicéntrica en aproximadamente
90\% de los casos, sin embargo, puede aparecer con forma multicéntrica como en nuestro caso que tenía linfadenopatías en región retroperitoneal adyacentes a la masa tumoral y en mediastino. La forma multicéntrica es agresiva y puede estar asociada a condiciones como síndrome POEMS, amiloidosis, sarcoma de Kaposi, insuficiencia renal e infección por VHH8 y VIH. Las localizaciones más frecuentes a nivel de la economía corporal en la EC son torácicas $(60 \%)$, cervical (14\%), abdominal (11\%) y axilar (4\%) (8). En nuestro caso específico encontramos la ubicación de la lesión tumoral prominente estaba en retroperitoneo, que no constituye una de las localizaciones más habituales, siendo aproximadamente el 3\%, pero el subtipo histológico encontrado fue HV que es el más frecuente en la mayoría de series revisadas como es el caso de Kawamura et al. (9) quienes informaron que subtipo HV se encontró en $87 \%$ de 132 casos de enfermedad de Castleman retroperitoneal en población japonesa.

Teniendo en cuenta que los elementos clínicos cardinales de nuestro caso se situaron a nivel de piel con el diagnóstico de PPN, cabe señalar que aproximadamente en $18 \%$ de los casos existe asociación de esta entidad con EC. No obstante, existe un número limitado de casos reportados de PPN asociado a EC (10). El PPN es una enfermedad mucocutánea autoinmune caracterizada por estomatitis severa, erupciones cutáneas polimórficas y neoplasias subyacentes asociadas, histopatológicamente caracterizada por la presencia de células epiteliales disqueratósicas, con acantólisis e inmunofluorescencia típica. El PPN se ha caracterizado por tener mal pronóstico con un rango de mortalidad de 75 - $90 \%$ y una supervivencia media de menos de un año (11). Para el diagnóstico de PPN se recomienda una combinación de criterios diagnósticos que incluyen erosiones dolorosas de la mucosa y erupciones polimórficas de la piel, características histopatológicas de acantólisis intraepidérmica, disqueratosis y dermatitis de interfase vacuolar; inmunofluorescencia directa 
con hallazgos de IgG epidérmica intercelular y complemento, anticuerpos séricos detectados por inmunofluorescencia indirecta que se unen a las células superficies de epitelios escamosos estratificados como también simple, columnar y transicional, finalmente inmunoprecipitación sérica con un complejo de cuatro proteínas (desmoplaquina I -250 kD-, antígeno del penfigoide ampollar-230 kD-, desmoplaquina II y envoplaquina $-210 \mathrm{kD}$-, periplaquina $-190 \mathrm{kD}$ ). Estos criterios diagnósticos fueron revisado posteriormente por Camisa y Helm quienes los dividieron en signos mayores y menores: los primeros incluyen erupción mucocutánea polimórfica, existencia de neoplasia concurrente y anticuerpos séricos con un específico patrón de inmunoprecipitación, mientras que los menores agrupan evidencia histológica de acantólisis, inmunofluorescencia directa mostrando tinción de membrana intercelular y basal, además estudio de inmunofluorescencia indirecta con epitelio de vejiga de rata. Tres criterios mayores, o dos mayores y dos menores se requieren para el diagnóstico de PPN. En el caso específico de nuestro paciente contamos con los tres criterios mayores y dos menores que sustentaron el diagnóstico realizado. El tratamiento más efectivo para el PNP es la resección o resolución de la neoplasia subyacente, como se ha demostrado cuando aparece asociado a la EC. La mortalidad por bronquiolitis obliterante que constituye la complicación más severa, es significativamente baja, posiblemente por extirpación temprana del tumor de Castleman y administración de inmunoglobulina $(11,12)$. Las altas dosis de corticosteroides sistémicos como prednisona (1.0-1.5 mg/ $\mathrm{kg} /$ día) e inmunosupresión con azatioprina y micofenolato para el tratamiento también se han recomendado, además el rituximab se ha propuesto dado a que actúa al reducir las células B responsables de producir anticuerpos causantes del desarrollo de lesiones en la piel (13). Aunque nuestro paciente recibió altas dosis de esteroides y un ciclo de terapia CHOP, no presentó mejoría de las lesiones cutáneo mucosas.

El tratamiento de la EC se dirige a la patogenia de la enfermedad sugerida. La escisión quirúrgica está indicada para la enfermedad unicéntrica ya sea la variante HV o de células plasmáticas, pero raramente emplea en la forma multicéntrica que es nuestro caso específico, mientras que la terapia citorreductora puede ser usada. Los regímenes quimioterápicos más frecuentes son el esquema CHOP que se administró a nuestro paciente, o la terapia con ciclofosfamida, doxorrubicina, vincristina y dexametasona (CAVD) (14). Algunos estudios han mostrado beneficios con la radioterapia y tratamiento inmunomodulador como esteroides, interferón alfa, ácido transtiretinoico y talidomida (14-16). Otros tratamientos son los anticuerpos monoclonales que incluyen aquellos con objetivo inhibir la interleuquina 6 como es el caso de siltuximab y altizumab, además el anti CD 20 rituximab (17). Aunque en nuestro paciente estaba indicado el uso de anticuerpo monoclonal para CD20, ya que era uno de los marcadores expresados en la inmunohistoquímica, no fue posible aplicar esta estrategia terapéutica debido falta de disponibilidad temporal en nuestro medio de rituximab por lo que se decidió asociar al tratamiento dosis de interferón y reducir la carga de terapia CHOP. También diversos fármacos antivirales como aciclovir, ganciclovir, cidofovir y valganciclovir han sido usados en el tratamiento de la viremia asociada a EC $(14,16,18)$.

El paciente durante su estancia en nuestra institución recibió seguimiento y tratamiento integral por grupo multidisciplinario; sin embargo, presentó celulitis en brazo derecho y debido al grado de inmunodepresión a pesar del tratamiento antimicrobiano de amplio espectro evolucionó ominosamente a sepsis posteriormente shock séptico y falleció durante su estancia en la unidad de cuidado intensivo polivalente.

\section{Conclusión}

Las lesiones mucocutáneas constituyen hallazgos clínicos frecuentes en la práctica médica, cuyo espectro etiológico comprende procesos infecciosos, enfermedades inmunológicas y entidades neoplásicas. Cuando son refractarias a tratamientos antimicrobianos e inmunosupresores debemos considerar que puedan ser parte del espectro clínico de un síndrome paraneoplásico. Se presentó el caso de un paciente con PPN asociado a enfermedad de Castleman de variante HV que usualmente tiene curso clínico benigno en la forma clínica unicéntrica; sin embargo, nuestro paciente padeció el tipo multicéntrico, con una evolución clínica ominosa, siendo la histopatología esencial para realizar diagnóstico.

\section{Referencias}

1. Bonekamp D, Horton KM, Hruban RH, Fishman EK. Castleman disease: the great mimic. Radiographics. 2011 Oct;31(6):1793-807. doi: 10.1148/ rg.316115502. PMID: 21997995.

2. Castleman B, Iverson L, Menendez VP. Localized mediastinal lymphnode hyperplasia resembling thymoma. Cancer. 1956 Jul-Aug;9(4):822-30. doi: 10.1002/1097-0142(195607/08)9:4<822::aid-cncr2820090430>3.0.co;2-4.PMID: 13356266.

3. Talat N, Belgaumkar AP, Schulte KM. Surgery in Castleman's disease: a systematic review of 404 published cases. Ann Surg. 2012 Apr;255(4):677-84. doi: 10.1097/SLA.0b013e318249dcdc. PMID: 22367441.

4. Szalat R,Munshi NC. Diagnosis of Castleman Disease. Hematol Oncol Clin North Am. 2018 Feb;32(1):53-64. doi: 10.1016/j.hoc.2017.09.005. PMID: 29157619.

5. Tirado-Sánchez A, Bonifaz A. Paraneoplastic Pemphigus. A Life-Threatening Autoimmune Blistering Disease. Actas Dermosifiliogr. 2017 Dec;108(10):902910. English, Spanish. doi: 10.1016/j.ad.2017.04.024. Epub 2017 Aug 8. PMID: 28801011.

6. Li Y, Zhao H, Su B, Yang C, Li S, Fu W. Primary hyaline vascular Castleman disease of the kidney: case report and literature review. Diagn Pathol. 2019 Aug 22;14(1):94. doi: 10.1186/s13000-019-0870-9. PMID: 31439011; PMCID: PMC6706926.

7. Zhang X, Rao H, Xu X, Li Z, Liao B, Wu H, Li M, Tong X, Li J, Cai Q. Clinica characteristics and outcomes of Castleman disease: A multicenter study of 185 Chinese patients. Cancer Sci. 2018 Jan;109(1):199-206. doi: 10.1111/cas.13439. Epub 2017 Dec 28. PMID: 29124835; PMCID: PMC5765290.

8. Erkurt MA, Aydogdu I, Kuku I, Kaya E, Mizrak B, Basaran Y. A multicentric hyaline vascular variant of Castleman's disease associated with B cell lymphoma: a case report. Cases J. 2009 Jun 10;2:8183. doi: 10.4076/1757-1626-2-8183. PMID: 19830060; PMCID: PMC2740273.

9. Shimokihara K, Kawahara T, Kasahara R, Kasuga J, Sugiura S, Tajiri R, Uemura H, Chiba K. Retroperitoneal Castleman's Disease. Case Rep Oncol. 2019 Nov 27;12(3):885-889. doi: 10.1159/000504700. PMID: 31911773; PMCID: PMC6940475.

10. Bin Waqar SH, Khan AA, Mohiuddin O, Rehan A. Paraneoplastic Pemphigus 
with Underlying Castleman's Disorder: A Rare Report with Literature Review. Cureus. 2019 Jun 28;11(6):e5022. doi: 10.7759/cureus.5022. PMID: 31501721; PMCID: PMC6721887.

11. Zhu X,Zhang B. Paraneoplastic pemphigus. J Dermatol. 2007 Aug;34(8):503-11. doi: 10.1111/j.1346-8138.2007.00322.x. PMID: 17683379.).

12. Paolino G, Didona D, Magliulo G, Iannella G, Didona B, Mercuri SR, Moliterni E, Donati M, Ciofalo A, Granata G, Ranuzzi P, Falasca V, Calvieri S. Paraneoplastic Pemphigus: Insight into the Autoimmune Pathogenesis, Clinical Features and Therapy. Int J Mol Sci. 2017 Nov 26;18(12):2532. doi: 10.3390/ ijms18122532. PMID: 29186863; PMCID: PMC5751135.

13. Maruta CW, Miyamoto D, Aoki V, Carvalho RGR, Cunha BM, Santi CG. Paraneoplastic pemphigus: a clinical, laboratorial, and therapeutic overview. An Bras Dermatol. 2019 Oct 17;94(4):388-398. doi: 10.1590/abd1806-4841.20199165. PMID: 31644609; PMCID: PMC7007015.

14. Casper C. The aetiology and management of Castleman disease at 50 years: translating pathophysiology to patient care. Br J Haematol. 2005 Apr;129(1):3-17. doi: 10.1111/j.1365-2141.2004.05311.x. PMID: 15801951.
15. Bowne WB, Lewis JJ, Filippa DA, Niesvizky R, Brooks AD, Burt ME, Brennan MF. The management of unicentric and multicentric Castleman's disease: a report of 16 cases and a review of the literature. Cancer. 1999 Feb 1;85(3):706-17. doi: 10.1002/(sici)1097-0142(19990201)85:3<706::aid-cncr21>3.0.co;2-7. PMID: 10091744 .

16. Soumerai JD, Sohani AR, Abramson JS. Diagnosis and management of Castleman disease. Cancer Control. 2014 Oct;21(4):266-78. doi: 10.1177/107327481402100403. PMID: 25310208.

17. Basnayake BMDB, Wazil AWM, Kannangara T, Ratnatunga NVI, Hewamana S, Ameer AM. Multicentric Castleman disease of hyaline vascular variant presenting with unusual systemic manifestations: a case report. J Med Case Rep. 2017 May 14;11(1):135. doi: 10.1186/s13256-017-1294-3.PMID: 28501028; PMCID: PMC5429940.

18. Chan KL, Lade S, Prince HM, Harrison SJ. Update and new approaches in the treatment of Castleman disease. J Blood Med.2016 Aug 3;7:145-58. doi: 10.2147/ JBM.S60514. PMID: 27536166; PMCID: PMC4976903. 